\title{
Energy transfer enhancement by oxygen perturbation of spin-forbidden electronic transitions in aromatic systems
}

\author{
A. Monguzzi, * R. Tubino, M. M. Salamone, and F. Meinardi \\ Dipartimento di Scienza dei Materiali, Universitá Milano-Bicocca, via Cozzi 53, I-20125 Milano, Italy
}

(Received 10 August 2010; published 15 September 2010)

\begin{abstract}
Triplet-triplet energy transfer in multicomponent organic systems is usually entirely ascribed to a Dextertype mechanism involving only short-range donor/acceptor interactions. We demonstrate that the presence of molecular oxygen introduces a perturbation to the electronic structure of one of the involved moieties which can induce a large increase in the spin-forbidden transition oscillator strength so that the otherwise negligible Förster contribution dominates the overall energy transfer rate.
\end{abstract}

DOI: $10.1103 /$ PhysRevB.82.125113

PACS number(s): 33.50.- j, 42.79.Nv

In the last few years multicomponent organic systems for sensitized up-conversion have been grabbing the headlines allowing to blueshift the light at power densities comparable with the solar irradiance. ${ }^{1-5}$ Therefore they are presently considered one of most promising ways to improve the efficiency of the incoming new generation solar cells. As a consequence there is actually a renewed interest about phenomena involving forbidden transitions in organic molecular systems since these processes play a crucial role in the sensitized up-conversion photoluminescence (PL). Not only the efficiency of these systems but also the performance of all the phosphorescent organic light emitting devices ${ }^{6-8}$ are usually controlled by the presence of molecular oxygen, which introduces significant quenching effects. For these reasons, understanding the interactions between oxygen and conjugated molecules is of essential importance.

It is well known that molecular oxygen $\mathrm{O}_{2}$ in its triplet ground state can induce the de-excitation of metastable triplet states trough several mechanisms including collisional quenching, energy transfer (ET) or photoinduced chemical reactions. ${ }^{9-11}$ Moreover, the interaction between $\mathrm{O}_{2}$ and aromatic moieties can raise the oscillator strength of the otherwise completely forbidden singlet to triplet $\left(S_{0} \rightleftharpoons T_{1}\right)$ transition. Thus, this effect has been used as a standard technique to obtain informations on the electronic structure of the triplet state of small oligocenes by direct measurements of the perturbed $S_{0} \rightarrow T_{1}$ one photon absorption in solution. ${ }^{12}$ No effects on the enhancement produced by oxygen on the phosphorescent $T_{1} \rightarrow S_{0}$ transition have been so far reported, and very little is known on its effects on the ET, where $\mathrm{O}_{2}$ has been considered a sort of excitation-carrier between donor and acceptor moieties, ${ }^{13}$ but not as the responsible of any enhancement of their ability to directly interact.

In the case of the sensitized up-conversion in bicomponent organic systems, a large triplet population triplet states is obtained on the up-converting moiety by ET from the light harvesting species, thus understanding the role of the $\mathrm{O}_{2}$ on the triplet-triplet ET becomes crucial. Aim of this Letter is the demonstration that the resonant energy transfer between organic molecules involving spin-forbidden transitions can be enhanced by the presence of molecular oxygen that raises the $T_{1} \rightarrow S_{0}$ transition dipole moment in moieties containing polycondensated aromatic rings.
The samples investigated are mixed solutions of $\mathrm{Pt}(\mathrm{II}) \mathrm{oc}-$ taethylporphyrin (PtOEP), acting as light absorber, and of 9,10 diphenylanthracene (DPA), which is known to have completely forbidden triplet to singlet transitions. Both molecules have been purchased by Sigma-Aldrich and used asis. PtOEP and PtOEP/DPA solutions in tetrahydrofuran (THF) have been prepared in a glove box under argon atmosphere with an oxygen concentration below than $1 \mathrm{ppm}$. The PL measurements have been carried out by exciting the samples with the second harmonic of a pulsed Nd:Yag laser at $2.33 \mathrm{eV}(532 \mathrm{~nm})$. CW measurements employed a Hamamatsu spectrometer C10083CA (bandpass $8 \mathrm{~nm}$ ) for the signal detection while the time-resolved ones have been done in photon counting mode with a Hamamatsu R943-02 photomultiplier coupled to an Ortec 9353 multichannel scaler. The overall time resolution was better than $50 \mathrm{~ns}$.

Sensitized up-conversion takes place through the following photophysical steps: (i) one photon absorption of light by a donor molecule (PtOEP) producing singlet excited states, (ii) population of triplet states through fast intersystem crossing (ISC), (iii) ET toward metastable triplet states of the acceptor molecule (DPA), and (iv) triplet-triplet annihilation (TTA) giving rise to high-energy singlet excited states of the acceptor moiety from which the up-converted emission takes place. These systems are usually prepared in a carefully controlled atmosphere to prevent the oxygen quenching of all the long living triplet states involved. However, the unusual high efficiency of the ET step always observed suggests also an active role for the interaction between the residual $\mathrm{O}_{2}$ and the aromatic molecules in addition to the ordinary collisional and photochemical processes.

The influence of paramagnetic molecules with $S_{z} \neq 0$ on spin-forbidden electronic transitions has been described in the early 1960s by Hoijtink, ${ }^{14,15}$ who demonstrated that the role of the pure magnetic interaction had been previously overestimated. ${ }^{16,17}$ Let's consider a ground state aromatic molecule as DPA and a paramagnetic molecule $P$ with a triplet ground state as $\mathrm{O}_{2}\left(S_{z}=1\right)$. During the lifetime of the collision complex, singlet and triplet electronic states of DPA are mixed thanks to the exchange interaction with $P .{ }^{14-16}$ By applying first-order perturbation theory it is possible to estimate the finite transition dipole moment of the spin forbidden transition $S_{0} \rightarrow T_{1}$ of DPA produced by the intensity borrowing from the allowed $S_{0} \rightarrow S_{1}$ transition. The oscillator 
strength $f$ for the lowest singlet-triplet transition in DPA under the influence of $\mathrm{O}_{2}$ becomes ${ }^{14}$

$$
f_{S_{0} \rightarrow T_{1}}=\left[\frac{H_{E X}}{E_{S_{1}}-E_{T_{1}}}\right]^{2} f_{S_{0} \rightarrow S_{1}},
$$

where $H_{E X}$ is the relevant matrix element for the exchange interaction. In oligocenes, the oscillator strength of the $S_{0}$ $\rightarrow T_{1}$ is so small that also a such perturbation can raise its value by several order of magnitude.

In order to verify if the nonvanishing oscillator strength of the $T_{1} \rightleftharpoons S_{0}$ in DPA may give an additional dipole-dipole contribution to the resonant ET, we analyzed all the processes controlling the sensitized up-conversion as a function of the oxygen concentration. The PtOEP/DPA system, when excited in the $Q$ absorption band of the porphyrin at $2.33 \mathrm{eV}$, shows two main photoluminescence peaks: (i) at about 2.83 $\mathrm{eV}$ originating from the blue up-converted DPA emission and (ii) at $1.92 \mathrm{eV}$ arising from the red residual PtOEP phosphorescence. In a recent paper, we modeled the kinetics of the sensitized up-conversion and obtained expressions for the intensity of blue and red emissions as a function of the photophysical parameters characterizing the employed molecules. ${ }^{18,19}$ By following the same approach, it is possible to give an expression for the ratio between the energy transfer rate in presence of oxygen $\left(\bar{k}_{\mathrm{ET}}\right)$ and in an oxygen free solution $\left(k_{\mathrm{ET}}\right)$. If the excitation power density is low enough to ensure that the TTA is not the main deactivation channel for the DPA excited triplet states, it is easy to demonstrate that

$$
\bar{k}_{\mathrm{ET}} / k_{\mathrm{ET}} \equiv \Gamma=[\underbrace{\frac{k_{r a d}^{D}}{\bar{k}_{\text {rad }}^{D}}}_{\Delta} \underbrace{\frac{\bar{k}_{T}^{A}}{k_{T}^{A}}}_{\Omega^{-1}} \underbrace{2}_{Z} \underbrace{\frac{k_{\exp }^{D}}{\bar{k}_{D}^{D}}} \underbrace{\bar{I}_{A} / \bar{I}_{D}}_{\Theta}]^{1 / 2}
$$

where $k_{\text {rad }}^{D}, k_{\text {exp }}^{D}$, and $I_{D}$ are the radiative decay rate, the experimentally measured decay rate $\left(k_{e x p}^{D}\right.$ is the sum of the decay rate of donor triplets in absence of acceptor $k_{T}^{D}$ and the transfer rate $k_{\mathrm{ET}}$ ), and the PL intensity of the PtOEP, respectively; $k_{T}^{A}$ and $I_{A}$ are the decay rates of the excited triplet states of the DPA, and its up-converted PL intensity. The signed (unsigned) quantities refer to oxygen contaminated (free) solutions. All the parameters in the right side of Eq. (2) can be experimentally measured. Since the PL intensity immediately after a pulsed excitation is equal to the number of excited states times the radiative decay rate, the ration between the time-resolved PL intensities at $t=0$ without and with oxygen gives $\Delta$. From the same time-resolved measurements it is possible to evaluate the donor decay rates and of consequence $Z$. The emitting DPA singlets are indirectly populated by TTA from the corresponding triplets, therefore their PL decay time coincides with twice the triplet lifetime that is necessary to calculate $\Omega$. Finally $\Theta$ is simply obtained from the time-integrated PL intensities of the different emissions.

The parameters defined in Eq. (2) have been measured as a function of the oxygen amount in solution, which has been increased by exposing the sample prepared in glow box to air

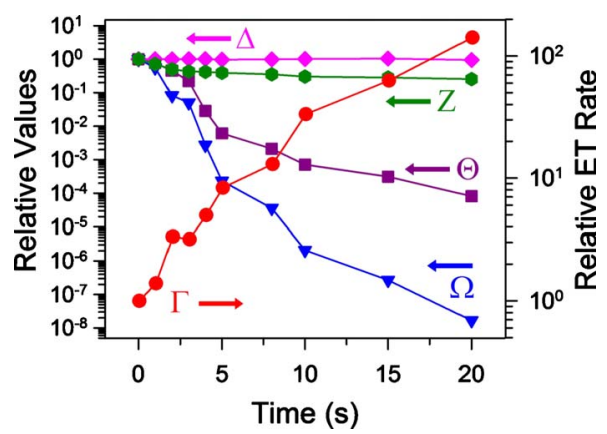

FIG. 1. (Color online) $\Delta, \Omega, Z, \Theta$, and $\Gamma$ experimental values for a PtOEP $\left(10^{-5} \mathrm{M}\right) / \mathrm{DPA}\left(3 \times 10^{-3} \mathrm{M}\right)$ solution $(\mathrm{THF})$, upon laser excitation at $2.33 \mathrm{eV}$, as a function of the $\mathrm{O}_{2}$ uptake.

for increasing times. All of them are sensitive to the $\mathrm{O}_{2}$, which is responsible of several quenching processes of both the donor and the acceptor excited states according to the $T_{1}+{ }^{3} \Sigma_{g}^{-}\left(\mathrm{O}_{2}\right) \rightarrow S_{0}+{ }^{1} \Delta_{g}\left(\mathrm{O}_{2}\right)$ reaction scheme. As a consequence, the triplet nonradiative decay rates $k_{T}^{D}$ and $k_{T}^{A}$ increase in presence of oxygen with negative consequences on the up-conversion yield regardless any possible effect on the transfer efficiency. ${ }^{9,10}$

Figure 1 reports the experimental values of $\Delta, \Omega, Z, \Theta$, and $\Gamma$ as a function of the exposition time to air. $\Delta$, that depends on the radiative decay rate of PtOEP $T_{1} \rightarrow S_{0}$ transition, is almost constant. This is not surprising since in this molecule, differently from the DPA case, the presence of an heavy atom enhances the spin-orbit coupling. ${ }^{18,20}$ Therefore the small extra contribution to the oscillator strength possibly coming from the interaction with the $\mathrm{O}_{2}$ is negligible.

The effect of the $\mathrm{O}_{2}$ on $Z$, i.e., on the PtOEP total decay rate, is only slightly larger than that on $\Delta$. In fact, since we are working with a large excess on DPA, in the oxygen-free solution $k_{\text {exp }}^{D}$ is very fast being mainly determined by the ET rate. The addition of $\mathrm{O}_{2}$ introduces a new nonradiative decay channel, which is however not so fast to compete significantly with the ET, at least in the investigated $\mathrm{O}_{2}$ concentration range.

On contrary, the presence of $\mathrm{O}_{2}$ strongly decreases the ratio between the up-converted blue PL and the residual PtOEP red emission inducing a reduction of $\Theta$ of about 4 order of magnitude. The overall up-conversion efficiency is therefore reduced because of the activation of nonradiative depopulation channels of the DPA triplet states, on which the up-conversion yield depends quadratically. This large quenching effect can be directly observed on $\Omega$ because: (i) the $T_{1} \rightarrow S_{0}$ is completely forbidden in DPA, resulting in a $k_{T}^{A}$ of only $800 \mathrm{~Hz}$ in the oxygen-free solution. The small oscillator strength induced by the interaction with the $\mathrm{O}_{2}$ is therefore very important and (ii) $\Omega$ depends on the square of the ratio $k_{T}^{A} / \bar{k}_{T}^{A}$.

Despite the sharp rise of DPA triplets decay rate, from the data reported in Fig. 1 it is evident that the oxygen-induced decrease in $I_{A}$ is less pronounced than expected. This can be explained only by assuming an enhancement of the ET rate in presence of $\mathrm{O}_{2}$, that partially counterbalance the quenching of the $T_{A}$ states. The $\Gamma$ values, i.e., the relative changes in 


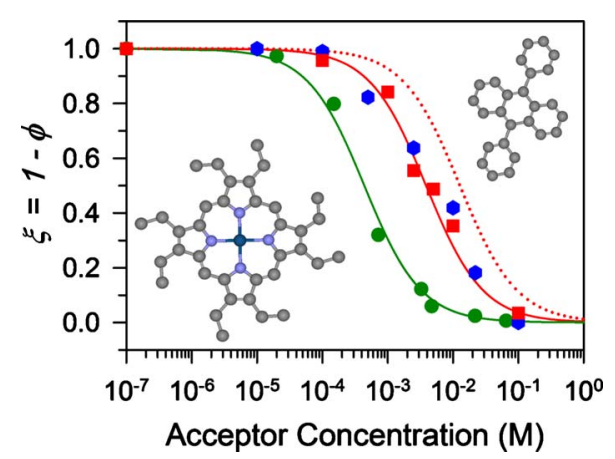

FIG. 2. (Color online) Relative emission quantum yield $\xi$ of PtOEP in THF solution $\left(10^{-5} \mathrm{M}\right)$ as a function of DPA concentration, in absence (dots) and in presence of $\mathrm{O}_{2}$ (squares and hexagons). Solid and dotted lines represent the best fit of the experimental data or have been computed as described in the text. Inset: molecular structures of PtOEP and DPA.

$k_{\mathrm{ET}}$ vs the $\mathrm{O}_{2}$ concentration calculated by Eq. (2), are reported in Fig. 1, which shows that the ET rate increases by a factor 100 in respect to its value in an oxygen-free solution. These findings unambiguously demonstrate that the resonant ET rate involving completely forbidden transitions of organic molecules can be enhanced by the exchange interaction between molecular oxygen and aromatic rings.

It is now possible to discuss the relative weight of the Dexter and Förster contributions to the overall ET in our typical multicomponent system for sensitized up-conversion in presence of $\mathrm{O}_{2}$. To this end we have investigated the $\mathrm{PtOEP}$ emission relative quantum yield $\xi$ as a function of the acceptor concentration for solutions both with and without oxygen. For a solution with a fixed oxygen concentration (in absence of the acceptor) the donor triplets decay rate $k_{T}^{D}$ is determined by radiative and nonradiative decay processes, including in these latter the oxygen quenching. The addition of an acceptor opens a new quenching channel for the donor through the ET, thus reducing $\xi=k_{T}^{D} /\left[k_{T}^{D}+k_{\mathrm{ET}}\right]$. Here $k_{\mathrm{ET}}$ is the total energy transfer rate given by the sum of the Dexter and Förster contribution $k_{\mathrm{ET}}=k_{\mathrm{ET}}^{\text {Dexter }}+k_{\mathrm{ET}}^{\text {Förster }}$.

Figure 2 shows the measured $\xi$ as a function of the acceptor concentration $\left(C_{\mathrm{DPA}}\right)$ for PtOEP $10^{-5} \mathrm{M}$ THF solutions. The reported values have been normalized to the PtOEP PL yield in absence of acceptors.

The analysis of these data must take into account for the direct donor-acceptor ET, as well as for the ET enhancement arising from the molecular diffusion. The relative weight of these two processes depends on the relative values of the diffusion length $D \tau_{0}$, (where $D$ is the total diffusion coefficient and $\tau_{0}$ is the excited donor lifetime in absence of any acceptors) and the square of the mean donor-acceptor distance $s^{2}$. Since we used a low viscosity solvent $(\eta=0.48 \mathrm{cP}$ at $300 \mathrm{~K})$ and small molecules (effective radius $R_{\text {eff }}^{D}=8.7 \AA, R_{\text {eff }}^{A}=4.5 \AA$ calculated accordingly with Ref. 21) which imply $D$ as large as $4.5 \times 10^{-5} \mathrm{~cm}^{2}$, having $\tau_{0}=\left(k_{T}^{D}\right)^{-1}=69.9 \mu \mathrm{s}$ the requirement for the so called rapid-diffusion limit is fulfilled. Indeed, even at the lowest acceptor concentration $\left(C_{\mathrm{DPA}}=2.5 \times 10^{-5} \mathrm{M}\right)$ at which $s=1.5 \times 10^{-5} \mathrm{~cm}, D \tau_{0} / s^{2} \approx 19 \gg 1$. In this regime all donors are identical, their PL decays are single exponential and they experience the maximum of ET efficiency. ${ }^{22-25}$

For the oxygen-free sample, since the DPA $T_{1} \rightleftharpoons S_{0}$ transition is completely forbidden, the long-range Förster contribution to the overall ET is negligible. Therefore this set of experimental data has been fitted by considering a pure Dexter interaction mechanism. In the rapid-diffusion limit, by using the Perrin ${ }^{26,27}$ approximation, $k_{\mathrm{ET}}^{\text {Dexter }}=4 \pi D C_{\mathrm{DPA}} R_{D X}$ being $R_{D X}$ the characteristic interaction distance. With a measured $k_{T}^{D}=1.43 \times 10^{4} \mathrm{~Hz}$, the best fit gives $R_{D X}=9.8 \AA$ a value very close to the sum of the PtOEP and DPA effective $\operatorname{radii}\left(R_{\text {eff }}^{T O T}=R_{\text {eff }}^{D}+R_{\text {eff }}^{A}=13.2 \AA\right)$ thus demonstrating that the donor and acceptor must come in close contact to make the ET effective. It is not surprising that the obtained $R_{D X}$ is slightly smaller than $R_{e f f}^{T O T}$ since in the calculation of the effective radius the molecules are considered spheres, with an overestimation of the minimum approaching distance for approximately flat molecules such as PtOEP and DPA.

The same measurements have been repeated after the exposition of the solutions to the air for few minutes. In this case $k_{T}^{D}$ increases about 30 times to $4.35 \times 10^{5} \mathrm{~Hz}$ as a consequence of the PtOEP quenching induced by the $\mathrm{O}_{2}$. The ET process becomes less efficient but its decrease is not as large as expected. Indeed, the theoretical dependence of $\xi$ on the DPA concentration calculated for $R_{D X}=9.8 \AA$ and $k_{T}^{D}=4.35$ $\times 10^{5} \mathrm{~Hz}$ (see Fig. 2 dotted line) is much more shifted toward large DPA concentrations than the measured value (Fig. 2 squares). Therefore, the experimental data have been fitted including also the contribution to the ET of a long-range Förster interaction which is, in the rapid-diffusion limit, $k_{\mathrm{ET}}^{\text {Förster }}=4 \pi C_{\mathrm{DPA}} R_{F S}^{6} k_{T}^{D} /\left(3 a^{3}\right)$, being $a$ the closest approach between donors and acceptors. ${ }^{22,23}$ By using for $a$ the $R_{D X}$ previously obtained, the best fit gives a Förster radius of $20.2 \AA$ (by using $a=R_{\text {eff }}^{T O T}=13.2 \AA R_{F S}$ increases only slightly to $23.3 \AA$ ). It should be noted that the plots of $\xi$ vs $C_{\mathrm{DPA}}$ reported in Fig. 2 do not allow to discriminate between Dexter and Förster contributions since in the rapid-diffusion limit both these processes imply the same linear dependence of $k_{\mathrm{ET}}$ on acceptor concentration. This explain the overestimation of $R_{D X}$ reported for a similar systems probably still containing traces of $\mathrm{O}_{2} \cdot{ }^{28}$

In order to verify if the observed ET rate behavior is given by an increase in the interaction distance and not to other quenching related effects, $\xi$ values as a function of the acceptor concentration have been also recalculated from the DPA up-converted PL by using the equation ${ }^{18}$

$$
I_{A} \propto\left[T_{A}\right]^{2}=\left(\frac{1}{\bar{k}_{T}^{A}}\right)^{2}\left(\frac{\bar{k}_{\mathrm{ET}}}{\bar{k}_{0}^{D}+\bar{k}_{\mathrm{ET}}}\right)^{2}=\left(\frac{1}{{\overline{k_{T}^{A}}}^{A}}\right)^{2}(1-\xi)^{2},
$$

As it is evident from the Fig. 2 (hexagons) these data are fully consistent with those obtained from the direct measurement of the PtOEP relative quantum efficiency, confirming the oxygen-induced ET enhancement.

In conclusion, it has been demonstrated that the ET between spin-forbidden transitions of organic donor/acceptor pairs can be enhanced by the presence of molecular oxygen, given by its exchange coupling with the acceptor molecule. 
During the lifetime of the collisional complex (acceptor) $+\mathrm{O}_{2}$, this interaction perturbs the acceptor electronic structure, generating a finite moment for its lowest spin forbidden $T_{1} \rightarrow S_{0}$ transition. The Förster-type contribution to the overall energy transfer becomes dominant at large $\mathrm{O}_{2}$ concentrations. This shed light on the anomalously large ET radius $\left(\begin{array}{ll}> & \AA\end{array}\right)$ previously observed in such family of systems involving polycondensated aromatic rings acting as acceptors.

This work has been supported by Fondazione Cariplo and by the research found "Dote ricercatori:" FSE, Regione Lombardia. *angelo.monguzzi@mater.unimib.it

${ }^{1}$ S. Baluschev, T. Miteva, V. Yakutkin, G. Nelles, A. Yasuda, and G. Wegner, Phys. Rev. Lett. 97, 143903 (2006).

${ }^{2}$ R. R. Islangulov, J. Lott, C. Weder, and F. N. Castellano, J. Am. Chem. Soc. 129, 12652 (2007).

${ }^{3}$ S. Baluschev, V. Yakutkin, T. Mitieva, G. Wegner, T. Roberts, G. Nelles, A. Yasuda, S. Chernov, S. Aleshchenkov, and A. Cheprakov, New J. Phys. 10, 013007 (2008).

${ }^{4}$ T. N. Singh-Rachford, J. Lott, C. Weder, and F. N. Castellano, J. Am. Chem. Soc. 131, 12007 (2009).

${ }^{5}$ A. Monguzzi, R. Tubino, and F. Meinardi, J. Phys. Chem. A 113, 1171 (2009)

${ }^{6}$ M. A. Baldo, C. Adachi, and S. R. Forrest, Phys. Rev. B 62, 10967 (2000).

${ }^{7}$ S. Reineke, K. Walzer, and K. Leo, Phys. Rev. B 75, 125328 (2007).

${ }^{8}$ N. C. Giebink and S. R. Forrest, Phys. Rev. B 77, 235215 (2008).

${ }^{9}$ A. P. Darmanyan, Chem. Phys. Lett. 91, 396 (1982).

${ }^{10}$ A. K. Bansal, W. Holzer, A. Penzkofer, and T. Tsuboi, Chem. Phys. 330, 118 (2006).

${ }^{11}$ Z. J. Diwu and J. W. Lown, J. Photochem. Photobiol., A 64, 273 (1992).

${ }^{12}$ J. S. Brinen and J. G. Koren, Chem. Phys. Lett. 2, 671 (1968).

${ }^{13}$ H. Kim, N. Dashdorj, H. M. Zhang, J. S. Yan, W. A. Cramer, and S. Savikhin, Biophys. J. 89, L28 (2005).
${ }^{14}$ G. J. Hoijtink, Mol. Phys. 3, 67 (1960).

${ }^{15}$ J. N. Murrell, Mol. Phys. 3, 319 (1960).

${ }^{16}$ Y. Chiu, J. Chem. Phys. 56, 4882 (1972).

${ }^{17}$ G. Porter and M. R. Wright, Discuss. Faraday Soc. 27, 18 (1959).

${ }^{18}$ A. Monguzzi, J. Mezyk, F. Scotognella, R. Tubino, and F. Meinardi, Phys. Rev. B 78, 195112 (2008).

${ }^{19}$ J. Mezyk, R. Tubino, A. Monguzzi, A. Mech, and F. Meinardi, Phys. Rev. Lett. 102, 087404 (2009).

${ }^{20}$ D. Beljonne, Z. Shuai, G. Pourtuois, and J. L. Bredas, J. Phys. Chem. A 105, 3899 (2001).

${ }^{21}$ A. Bondi, J. Phys. Chem. 68, 441 (1964).

${ }^{22}$ D. D. Thomas, W. F. Carlsen, and L. Stryer, Proc. Natl. Acad. Sci. U.S.A. 75, 5746 (1978).

${ }^{23}$ L. Stryer, D. D. Thomas, and C. F. Meares, Annu. Rev. Biophys. Bioeng. 11, 203 (1982).

${ }^{24}$ I. Z. Steinberg and E. Katchalski, J. Chem. Phys. 48, 2404 (1968).

${ }^{25}$ B. P. Lyons and A. P. Monkman, Phys. Rev. B 71, 235201 (2005).

${ }^{26}$ F. Perrin, Compt. Rend. 178, 1978 (1924).

${ }^{27}$ U. Gösele, M. Hauser, U. K. A. Klein, and R. Frey, Chem. Phys. Lett. 34, 519 (1975).

${ }^{28}$ A. Monguzzi, R. Tubino, and F. Meinardi, Phys. Rev. B 77, 155122 (2008). 